\title{
Equilíbrio postural e risco de quedas de idosas praticantes do método pilates e idosas sedentárias
}

Postural balance and risk of falling in elderly women who practice pilates method and those who are sedentary

FisiSenectus. Unochapecó Ano 5, n. 1 - Jan/Jun. 2017 p. $42-49$

Ana Paula Karolczak. akarolczak@unisinos.br

Fisioterapeuta. Mestre em Ciências do Movimento Humano. Docente do Curso de Fisioterapia da Universidade do Vale do Rio dos Sinos - UNISINOS.

Tissiani Morimoto. tissi.morimoto@gmail.com

Fisioterapeuta. Mestre em Saúde Coletiva. Docente do Curso de Fisioterapia do Centro Universitário Ritter dos Reis - UNIRITTER.

Carolina Dacroce Mantovani. caroldm_mantovani@hotmail.com

Fisioterapeuta.

\section{Resumo}

Introdução: O envelhecimento é um processo natural na vida do ser humano, no qual ocorrem modificações nos sistemas fisiológicos e na capacidade funcional, afetando assim, a qualidade de vida do idoso. Desta forma, a fisioterapia tem um papel importante na prevenção e na reabilitação, visando sempre o tratamento mais adequado, para diminuir e/ ou evitar incapacidades, e/ou melhorar a qualidade de vida do idoso. Objetivo: Comparar o equilíbrio estático e dinâmico, o risco de quedas e a coordenação motora de idosas praticantes de Pilates e idosas sedentárias. Materiais e métodos: A coleta de dados foi realizada a partir de um questionário, no qual continha questões sobre dados demográficos e comportamentais. 0 equilíbrio e o risco de quedas foram avaliados a partir da Escala de equilíbrio de Berg e do teste Timed Up and Go, respectivamente, e foram realizados testes de coordenação motora de membros inferiores. Os dados foram analisados no programa SPSS versão 21.0 e foi considerado $p<0,05$ como nível de significância. Participaram do estudo 30 indivíduos, todos do sexo feminino, sendo 15 pertencentes ao grupo controle e 15 ao grupo experimental. A média de idade no grupo controle foi de $67,6 \pm 3,71$ anos e no grupo experimental foi de $67,53 \pm 3,6$ anos. Conclusão: Todos os testes realizados com 0 grupo experimental apresentaram resultados significativos quando comparados ao grupo controle. Os resultados deste estudo apontam que a prática do método Pilates por idosas melhora/preserva o equilíbrio dinâmico e estático e a coordenação motora, e diminui o risco de quedas.

\section{Palavras-chave}

Fisioterapia; Equilíbrio postural; Acidentes por quedas; Envelhecimento; Método Pilates.

\section{Fisißenectus}




\begin{abstract}
Introduction: Aging is a natural process in human life, in which there are changes in physiological systems and functional capacity, thus affecting the quality of life of the elderly. This way, the therapy plays an important role in prevention and rehabilitation, always seeking the most appropriate treatment to reduce and / or avoid disability, and / or improve the quality of life of the elderly. Objective: This study aimed to compare the static and dynamic balance, the risk of falling and motor skills in elderly women who practice Pilates method and those who are sedentary. Materials and Methods: The data were collected by means a questionnaire, in which included questions about demographic and behavioral data. The balance and the risk of falling were evaluated from the Berg Balance Scale and the Timed Up and Go test, respectively, and the coordination movement tests were performed on the lower limbs. The data were analyzed in SPSS software, version 21.0 and it was considered $p<0.05$ such as significance level. The study included 30 patients, all female, 15 in the control group and 15 to the experimental group. The average age in the control group was $67.6 \pm 3.71$ years and the experimental group was $67.53 \pm 3.6$ years. Conclusion: All tests with the experimental group had meaningful results when compared to the control group. The results of this study indicate that the practice of Pilates method by elderly women improve/ preserve the dynamic and static balance and motor coordination, and reduces the risk of falling.
\end{abstract}

\title{
Keywords
}

Physiotherapy; Postural balance; Falling accidents; Aging; Exercise and movement techniques.

\section{Introdução}

$\infty \infty \infty \times \infty \times \infty \times \infty \times \infty \times \infty \times \infty \times \infty \times \infty \times \infty \times \infty \infty \infty$

0 aumento da expectativa de vida vem crescendo mundialmente nos últimos anos, e novas pesquisas clínicas e estratégias em relação às questões de saúde pública relacionadas ao enveIhecimento vêm sendo desenvolvidas ${ }^{1}$. A cada ano, a população brasileira incorpora 700 mil novos idosos, a maioria com doenças crônicas e alguns com limitações funcionais². Com isso, qualquer situação destinada aos idosos deve levar em conta a capacidade funcional, a necessidade de autonomia, de participação, de cuidado e de auto-satisfação, e, principalmente, a prevenção, o cuidado e a atenção integral à saúde.

0 processo de envelhecimento está associado a diminuição da força, da massa muscular e do equilíbrio. Sabe-se que uma das principais características marcantes do processo de envelhecimento, que limita a vida do idoso, é o desequilíbrio ${ }^{3}$. A instabilidade postural, devido ao desequilíbrio, é um dos fatores que causam limitações nas atividades de vida diária do idoso. As consequências mais danosas dessa instabilidade são as quedas, que acabam limitando a mobilidade funcional e, com isso, torna os idosos mais dependentes ${ }^{4}$.
Além disso, a coordenação motora no idoso previne o risco de quedas ${ }^{5}$. Quando afetada, o idoso possui maiores dificuldades nas atividades do cotidiano que exigem uma boa coordenação, como combinar movimentos, levantar-se da cadeira, desviar de obstáculos e pessoas, andar rapidamente pela casa para atender ao telefone, e atravessar a rua em uma velocidade segura, fazendo com que o risco de quedas aumente durante essas atividades.

No Brasil, cerca de $29 \%$ dos idosos sofreram quedas no período de um $a n o^{6}$. Karuka, Silva e Navega $^{7}$ afirmam que as quedas são 0 acidente mais comum entre os idosos e têm grande importância social, epidemiológica e econômica, mundialmente. As quedas podem causar sérios riscos, como diminuição das atividades motoras, depressão, isolamento social, institucionalização, dependência em algumas atividades básicas funcionais, redução da qualidade de vida e, até mesmo, a morte.

Tendo em vista esses dados, percebe-se a importância de um tratamento que contemple a prevenção de quedas em idosos. Nesse sentido, Rossi et al. ${ }^{8}$ relatam a importância do fisioterapeuta em conhecer o idoso para desenvolver um planejamento, seja de reabilitação ou de prevenção, a fim 
de diminuir alguma dependência e incapacidade e/ou melhorar a qualidade de vida desse idoso.

Dentre as técnicas que o fisioterapeuta pode eleger, o método Pilates tem sido bastante utilizado. 0 Pilates inseriu-se entre os métodos utilizados pelos profissionais de saúde com o objetivo de integrar a mente e o corpo, refletindo na meIhora da flexibilidade, da força, do equilíbrio e da consciência corporal9. A prática de exercícios por meio deste método é uma possibilidade de tratamento e prevenção, pois é composto de exercícios que utilizam contrações isotônicas e isométricas, enfatizando o que seu criador Joseph $\mathrm{H}$. Pilates denominou de power house, que se trata de um complexo constituído pelos músculos abdominais, paravertebrais lombares e glúteos, responsáveis pela estabilização estática e dinâmica do corpo ${ }^{10}$.

Nesse contexto, o objetivo do presente estudo foi comparar o equilíbrio estático e dinâmico, a coordenação motora, e o risco de quedas em idosas praticantes de Pilates.

\section{Metodologia}

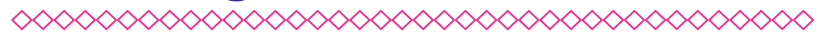

Este estudo é caracterizado como observacional descritivo do tipo transversal, e foi aprovado pelo Comitê de Ética em Pesquisa da Universidade, sob o parecer $n^{\circ}$ CEP 14/204. Todos os participantes leram e assinaram o Termo de Consentimento Livre e Esclarecido (TCLE).

A amostra foi composta por mulheres idosas praticantes de Pilates em um Estúdio da cidade de Esteio/RS, e mulheres idosas sedentárias, ou seja, que praticavam menos de 150 minutos de atividade física por semana ${ }^{11}$ da mesma cidade e conhecidas das idosas praticantes de Pilates, compondo, respectivamente, o grupo experimental e controle. Todas as participantes deveriam ser do sexo feminino e ter idade entre 60 e 80 anos. As participantes do grupo experimental deveriam apresentar frequência de prática de pelo menos duas vezes na semana, há pelo menos seis meses. Foram excluídas as idosas que realizavam outras atividades físicas e que apresentaram alterações cognitivas que impossibilitavam a realização dos testes.
A coleta de dados ocorreu entre os dias 10 de março e 15 de abril de 2015. Foram coletados dados sociodemográficos como idade, sexo, atividades físicas praticadas e patologias associadas. Após, foi realizado o teste Mini Mental ${ }^{12}$ para avaliar presença de alterações cognitivas.

As participantes que estavam de acordo com os critérios de inclusão realizaram primeiramente a Escala de Equilíbrio de Berg ${ }^{13}$ com o objetivo de avaliar o equilíbrio estático e dinâmico, tendo como pontuação máxima de 56 pontos e quanto maior a pontuação, melhor o equilíbrio. Para avaliar o risco de quedas, foi realizado o Teste Timed Up and Go (TUG) ${ }^{7}$, cujo resultado é pontuado em segundos. 0 teste realizado em até 10 segundos é o tempo considerado normal para adultos saudáveis, independentes e sem risco de quedas, entre 11 e 20 segundos é o esperado para idosos com deficiência ou frágeis, com independência parcial e com baixo risco de quedas, e o tempo acima de 20 segundos indica déficit importante da mobilidade física e risco de quedas. Posteriormente, os seguintes testes para avaliar a coordenação motora de membros inferiores foram realizados: ficar em pé sobre um pé só, ficar em pé de olhos fechados, caminhar com um pé na frente do outro, andar ao redor de obstáculos e subir escadas sem corrimão. Estes testes foram graduados em uma escala de um a cinco, sendo um, atividade impossível, e cinco, desempenho normal ${ }^{14}$.

Os dados obtidos foram organizados em um banco de dados para que a análise da estatística descritiva fosse realizada. Para a apresentação das variáveis numéricas foram utilizadas as medidas de tendência central e de dispersão, e para as variáveis categóricas foram utilizadas as medidas de frequências absolutas e relativas. 0 Teste de Levene foi utilizado para verificar a homogeneidade das variâncias, e o teste de Shapiro-Wilk para avaliação da normalidade dos dados. Para comparação da distribuição das médias obtidas entre os grupos foi utilizado o teste $U$ de Mann-Whitney. Foi considerado $p<0,05$ como nível de significância. Todas as análises foram realizadas no programa SPSS versão 21.0 . 


\section{Resultados}

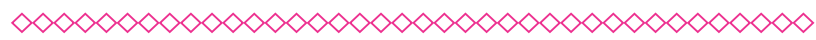

Participaram deste estudo 30 idosas do sexo feminino, sendo 15 do grupo controle e 15 do grupo experimental. A média de idade no grupo controle foi de $67,6 \pm 3,71$ anos e no grupo experimental $67,53 \pm 3,6$ anos $(p=0,961)$. Em relação ao estado cognitivo, nenhuma das participantes apresentou déficit, tendo como resultado do teste Mini Mental a média de $28,46 \pm 0,83$ pontos para o grupo controle e $28,8 \pm 0,67$ pontos para o grupo experimental $(p=0,239)$. No grupo experimental, a média de frequência semanal de prática de Pilates foi de $2,2 \pm 0,41$ vezes na semana e o tempo médio de prática foi de $3,6 \pm 1,88$ anos.

A Tabela 1 apresenta os resultados relacionados aos testes de equilíbrio, risco de quedas e coordenação motora realizados com as participantes do estudo. É possível observar que todos os testes realizados apresentaram resultados significativos para o grupo experimental, indicando melhor equilíbrio estático e dinâmico, menor índice de risco de quedas e melhor coordenação motora para as idosas praticantes do método Pilates.

\section{Discussão}

$\infty \times \infty \times \infty \times \infty \times \infty \times \infty \times \infty \times \infty \times \infty \times \infty \times \infty \times \infty \infty \infty$

A prevalência de queixas de desequilíbrio na população idosa chega a $85 \%$, podendo estar associada a diversas etiologias e causar desvios na marcha, instabilidade, vertigens e quedas frequentes $^{15}$, sendo assim, a manutenção do equilíbrio é fundamental para manter a independência funcional do idoso. Neste contexto, o presente estudo buscou avaliar o equilíbrio estático e dinâmico, risco de quedas, e a coordenação motora de idosas praticantes de Pilates.

No que se refere à amostra do estudo, observou-se que os grupos apresentaram características semelhantes, com a média de idade de ambos os grupos estatisticamente igual, assim como no teste Mini Mental. Os resultados do Mini Mental encontrados em ambos os grupos deste estudo estão de acordo com o valor esperado tanto para adultos jovens quanto para idosos ${ }^{16}$. Dessa forma, os grupos apresentaram um bom nível cognitivo geral, que é um fator positivo para os idosos, visto que com o processo de envelhecimento é comum a redução das funções cognitivas ${ }^{1}$.

No presente estudo pôde-se observar que as idosas praticantes de Pilates obtiveram melhor escore na Escala de Berg, indicando melhor equilíbrio deste grupo. Surbala et al. ${ }^{17}$, em um estudo cujo objetivo foi comparar a eficácia da intervenção de Pilates com um treinamento de equilíbrio no equilíbrio e na qualidade de vida em idosos, encontraram resultados que corroboram com o presente estudo. Os autores observaram melhora significativa no equilíbrio dos idosos praticantes de Pilates quando comparados aos outros grupos do estudo.

Da mesma forma, Pestana et al. ${ }^{18}$ realizaram um estudo com o objetivo de verificar e comparar os efeitos sobre a marcha e o equilíbrio de idosos após intervenção, utilizando dois métodos diferentes (Pilates e exercício resistido). A amostra foi constituída por 78 idosos, divididos em dois grupos: grupo experimental (Grupo Pilates: $n=39$ ) e grupo controle ativo (Grupo Exercício Resistido: $\mathrm{n}=39$ ). A intervenção com as duas técnicas ocorreu durante um período de 20 semanas. Os resultados do estudo revelaram que houve melhora estatisticamente significante sobre o equilíbrio dos idosos após a intervenção, para ambos os grupos, demonstrando, assim, que os dois métodos podem ser aplicados com a finalidade de potencializar a força muscular, contribuindo para a melhora do equilíbrio postural.

A melhora do equilíbrio pode ser justificada analisando o estudo de Marshall e Murphy ${ }^{19}$, no qual atribuem a melhora do equilíbrio à estabilização postural. Para garantir essa estabilização, é preciso reforço dos músculos posturais, principalmente os abdominais e os paravertebrais que, segundo Joseph Pilates, são o centro do corpo ${ }^{20}$.

O principal risco do déficit de equilíbrio em idosos é a ocorrência de quedas ${ }^{5}$. Alves e Scheicher ${ }^{21}$ relatam que a queda é um evento inesperado e não intencional que ocorre devido à perda do equilíbrio postural e da ineficiência dos mecanismos necessários à manutenção do controle postural. Sendo assim, o ganho de equilíbrio diminui o risco de quedas em idosos e, consequentemente, promove sua saúde ${ }^{5}$. 
As idosas praticantes de Pilates também apresentaram melhor coordenação motora quando comparadas às idosas sedentárias. A melhora da coordenação motora pode ser devido ao fato dos exercícios de Pilates serem baseados em uma abordagem holística, que requerem ativação e coordenação de vários grupos musculares ao mesmo tempo, utilizando uma combinação de fortalecimento muscular, alongamento e respiração para desenvolver os músculos do tronco e restaurar o equilíbrio muscular ${ }^{22}$.

No estudo de Oliveira et al. ${ }^{23}, 12$ idosas foram avaliadas antes e após 18 sessões de Pilates. Entre as avaliações estavam testes que de coordenação motora como levantar-se da posição sentada, levantar-se da posição decúbito ventral, levantar-se da cadeira, locomover-se pela casa e vestir e tirar uma camiseta. Após as intervenções, os autores observaram melhora significativa da coordenação, com exceção do teste de vestir e tirar uma camiseta, que pode ser justificado pela escolha do protocolo de exercícios selecionado, em que apresentava apenas um exercício que exigia movimentos de agilidade e coordenação de membros superiores.

No presente estudo foi possível observar que o grupo de idosas praticantes de Pilates apresentou menor tempo no TUG comparado ao grupo das idosas sedentárias, que apresentaram resultado de independência parcial e baixo risco de quedas. De acordo com Dallasta e Radünz ${ }^{24}$, o Pilates favorece o ganho de flexibilidade e o fortalecimento dos músculos do centro do corpo, que garantem a estabilização corporal durante atividades estáticas e dinâmicas, além de favorecer o equilíbrio do praticante.

Silva, Almeida, Cassilhas, Cohen, Peccin, Tufik et al. ${ }^{25}$ avaliaram 61 idosos, sendo 39 pertencentes ao grupo experimental (exercícios resistidos com carga progressiva), e 22 ao grupo controle (exercícios sem carga). Os autores observaram que o grupo experimental apresentou diminuição no tempo do TUG, o que é um fator positivo pois este teste tem relação com o equilíbrio, a velocidade da marcha e a capacidade funcional, ou seja, fatores que estão relacionados com o risco de quedas. Apesar do método de intervenção ser diferente, os dados resultantes são semelhantes ao presente estudo, uma vez que o método Pilates também se trata de um tipo de exercício resistido. Segundo Câmara, Santarém, Wolosker e Dias ${ }^{26}$, os exercícios resistidos com carga consistem na realização de contrações de grupos musculares específicos contra alguma forma de resistência externa (pesos livres, máquinas e bandas elásticas), tendo como principal vantagem o adequado controle de todas as variáveis do movimento (posição e postura, velocidade de execução, amplitude do movimento, volume e intensidade). Além disso, os equipamentos utilizados para a realização dos exercícios resistidos permitem a regulagem das sobrecargas a serem utilizadas de acordo com o nível de aptidão do indivíduo. No Pilates acontecem contrações concêntricas, excêntricas e principalmente isométricas, enfatizando o grupo muscular denominado power house. E para a execução correta dos movimentos, é necessário habilidades como, por exemplo, fluidez de movimento, controle e precisão ${ }^{27,28}$.

As idosas sedentárias do estudo apresentaram menor equilíbrio estático e dinâmico e maior risco de quedas em relação às praticantes de Pilates. Sabe-se que o sedentarismo aumenta o risco de quedas no idoso limitando sua independência nas atividades de vida diária. Com isso, frequentemente, estudos relatam sobre a importância da prática de algum exercício, prevenindo assim as quedas e os impactos causados por elas ${ }^{29}$. Estes resultados estão de acordo com o estudo de Guimarães, Galdino, Martins, Vitorino, Pereira e Carvalho ${ }^{30}$, que avaliaram a mobilidade funcional de idosos ativos e idosos sedentários por meio do teste TUG. Concluíram que os idosos que praticavam exercícios levaram menor tempo para realizar o teste quando comparados aos idosos sedentários.

\section{Conclusão}

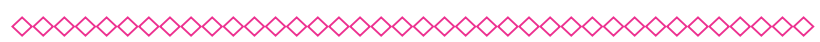

No presente estudo foi possível observar que as idosas praticantes do método Pilates apresentaram melhor equilíbrio, menor risco de quedas e melhor coordenação motora de membros inferiores quando comparadas às idosas sedentárias.

Portanto, percebe-se a importância de ações de promoção e prevenção conduzidas por fisioterapeutas, a fim de melhorar e/ou preservar o 
equilíbrio e a coordenação motora e diminuir o risco de quedas e a dependência e incapacidade em idosos.

Estudos futuros com amostras maiores poderão contribuir para melhor avaliar as tendências aqui relatadas, uma vez que o equilíbrio deve ser questão norteadora na saúde do idoso.

\section{Referências}

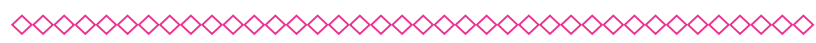

1. Altermann CDC, Martins AS, Carpes FP, Mello-Carpes PB. Influência da prática mental e observação do movimento sobre a memória motora, função cognitiva e desempenho motor em idosos. Braz J Phys Ther 2014;18(2):201-9.

2. Veras RP. Estratégias para o enfrentamento das doenças crônicas: um modelo em que todos ganham. Rev Bras Geriatr e Gerontol 2011;14(4):779-86.

3. Hernandes NA, Probst VS, Silva Jr RA, Januário RSB, Pitta F, Teixeira DC. Physical activity in daily life in physically independent elderly participating in community-based exercise program. Rev Bras Fisioter 2013;17(1):57-63.

4. Avelar NCP, Bastone AC, Alcântara MA, Gomes WF. Influência da prática mental e observação do movimento sobre a memória motora, função cognitiva e desempenho motor em idosos. Rev Bras Fisioter 2010;14(3):229-36.

5. Gomes ARL, Campos MS, Mendes MRP, Moussa L. A influência da fisioterapia, com exercícios de equilíbrio, na prevenção de quedas em idosos. FisiSenectus 2016;4(1):4-11.

6. Silva A Da, Faleiros HH, Shimizu WAL, Nogueira LDM, Nhãn LL, Silva BMF Da, et al. Prevalência de quedas e de fatores associados em idosos segundo etnia. Cien Saude Colet 2012;17(8):2181-90.

7. Karuka AH, Silva JAMG, Navega MT. Análise da concordância entre instrumentos de avaliação do equilíbrio corporal em idosos. Rev Bras Fisioter 2011;15(6):460-6.
8. Rossi ALS, Pereira VS, Driusso P, Rebelatto JR, Ricci $\mathrm{N}$ a. Profile of the elderly in physical therapy and its relation to functional disability. Brazilian J Phys Ther 2013;17(1):77-85.

9. Loss JF, Melo MO, Rosa CH, Santos AB, La Torre $M$, Silva YO. Atividade elétrica dos músculos oblíquos externos e multífidos durante o exercício de flexoextensão do quadril realizado no Cadillac com diferentes regulagens de mola e posições do indivíduo. Rev Bras Fisioter 2010;14(6):510-7.

10. Ferreira CB, Aidar FJ, Novaes GS, Vianna JM, Carneiro AL, Menezes LS. 0 método Pilates ${ }^{\circledR}$ sobre a resistência muscular localizada em mulheres. Motricidade 2007;3(4):76-81.

11. Lima DF, Luiz OC. Atividade física na promoção da saúde: uma avaliação das diretrizes. Semin Ciências Biológicas e da Saúde 2015;36(2):57-66.

12. Folstein MF, Folstein SE, McHugh PR. "Minimental state". A practical method for grading the cognitive state of patients for the clinician. J Psychiatr Res 1975;12:189-98.

13. Miyamoto ST, Lombardi I, Berg KO, Ramos LR, Natour J. Brazilian version of the Berg balance scale. Brazilian J Med Biol Res 2004;37(9): 1411-21.

14. O'Sullivan SB, Schmitz TJ. Fisioterapia: avaliação e tratamento. Barueri: Manole; 2010.

15. Carvalho EMS, Mota SPF, Silva GPF, Filho JMC. A postura do idoso e suas implicações clínicas. Geriatr Gerontol 2011;5(3):170-4.

16. Lourenço RA, Veras RP. Mini-Exame do Estado Mental: características psicométricas em idosos ambulatoriais. Rev Saúde Pública [Internet]. 2006;40(4):712-9.

17. Surbala L, Khuman PR, Trivedi P, Devanshi $B$, Mital V. Pilates versus Conventional Balance Training on Functional Balance and Quality of Life in Elderly Individuals: A Randomized Controlled Study. Sch J App Med Sci 2014;2(1B):221-6.

18. Pestana MCS, Pestana VS, Pestana AMS, Schinoni MI. Comparação entre os exercícios baseados no pilates solo versus exercício resistido 
sobre a marcha e equilíbrio do idoso. Rev Ciênc Méd Biol 2013;12(especial):441-8.

19. Marshall PW, Murphy BA. Core Stability Exercises On and Off a Swiss Ball. Arch Phys Med Rehabil 2005;86(1):242-9.

20. Endleman I, Critchley DJ. Transversus abdominis and obliquus internus activity during pilates exercises: measurement with ultrasound scanning. Arch Phys Med Rehabil 2008;89(11):2205-12.

21. Alves NB, Scheicher ME. Equilíbrio postural e risco para queda em idosos da cidade de Garça. Rev Bras Geriatr Gerontol 2011;14(4):763-8.

22. Irez GB, Ozdemir RA, Evin R, Irez SG, Korkusuz $F$. Integrating pilates exercise into an exercise program for $65+$ year-old women to reduce falls. J Sport Sci Med 2011;10(1):105-11.

23. Oliveira LC, Oliveira RF, Oliveira RG, Ganzella AC, Oliveira DAAP. Efeitos do método Pilates na autonomia funcional de idosas fisicamente ativas. Ter Man 2013;11(53):395-400.

24. Dallasta VC, Radünz RL. O Pilates na reabilitação fisioterapêutica em idosos: uma revisão. Uningá Rev 2013;15(1):44-7.

25. Silva A, Almeida GJM, Cassilhas RC, Cohen M, Peccin MS, Tufik S, et al. Equilíbrio, coordenação e agilidade de idosos submetidos à prática de exercícios físicos resistidos. Rev Bras Med do Esporte 2008;14(2):88-93.

26. Câmara LC, Santarém JM, Wolosker N, Dias RMR. Exercícios resistidos terapêuticos para indivíduos com doença arterial obstrutiva periférica: evidências para a prescrição. J Vasc Bras 2007;6(3):247-57.

27. Surbala L, Khuman PR, S GN, Kalpesh S. Pilates in Functional Balance and Quality of life in subacute stroke subjects - a randomized controlled study. Int J Heal Rehabil Sci 2013;2(4):204-11.

28. Sinzato CR, Taciro C, Pio CA, Toledo AM, Cardoso JR, Carregaro RL. Efeitos de 20 sessões do método Pilates no alinhamento postural e flexibilidade de mulheres jovens: estudo piloto. Fisioter Pesq 2013;20(2):143-50.

29. Fernandes AMBL, Ferreira JJA, Stolt LROG, Brito GEG, Clementino ACCR, Sousa NM. Efeitos da prática de exercício físico sobre o desempenho da marcha e da mobilidade funcional em idosos. Fisioter Mov 2012;25(4):821-30.

30. Guimarães LHCT, Galdino DCA, Martins FLM, Vitorino DFM, Pereira KL, Carvalho EM. Comparação da propensão de quedas entre idosos que praticam atividade física e idosos sedentários. Rev Neurociências 2004;12(2):68-72. 


\section{Anexos}

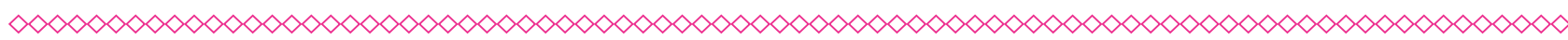

Tabela 1 - Valores da média e do desvio padrão para os testes de equilíbrio, risco de quedas e coordenação motora, em ambos os grupos avaliados (Esteio/RS, 2015). n em cada grupo=15.

\begin{tabular}{lccc} 
& Grupo controle & Grupo Experimental & $p$ \\
Berg (pontos) & $49,53 \pm 2,29$ & $54,53 \pm 0,83$ & $<0,01$ \\
TUG (segundos) & $13,53 \pm 1,59$ & $9,93 \pm 0,59$ & $<0,01$ \\
Um pé só (grau) & $3,46 \pm 0,51$ & $4,73 \pm 0,45$ & $<0,01$ \\
Olhos fechados (grau) & $3,86 \pm 0,63$ & $5 \pm 0$ & $<0,01$ \\
Pé na frente do outro (grau) & $3,53 \pm 0,51$ & $4,86 \pm 0,35$ & $<0,01$ \\
Obstáculos (grau) & $3,66 \pm 0,48$ & $5 \pm 0$ & $<0,01$ \\
Escada sem corrimão (grau) & $3,33 \pm 0,48$ & $4,33 \pm 0,48$ & $<0,01$ \\
\hline
\end{tabular}

Fonte: elaborado pelas autoras 